\title{
Outbreak of non-tuberculous mycobacteria in a paediatric bone marrow transplant unit associated with water contamination of needle-free connectors and literature review
}

\author{
Stephanie Habermann $\mathbb{D}^{1} \cdot$ Elena Ferran ${ }^{1} \cdot$ James Hatcher $^{2} \cdot$ Hermione Lyall $^{1} \cdot$ Josu De La Fuente $\mathbb{D}^{1} \cdot$ \\ Elizabeth Whittaker ${ }^{1}$
}

Received: 14 May 2020 / Revised: 9 January 2021 / Accepted: 3 February 2021 / Published online: 23 June 2021

(c) The Author(s) 2021. This article is published with open access

\section{To the Editor:}

Non-tuberculous mycobacteria (NTM) are ubiquitous acidfast bacteria existing in soil and water. They are divided into rapidly growing mycobacteria (RGM), which generates colonies on solid culture media within 7 days of incubation and slowly growing species which take weeks to grow. NTM lung disease is the most common clinical presentation of NTM disease. However, the clinical presentation of NTM systemic infections is often nonspecific and include fever, weight loss and lymphadenopathy. Disseminated disease may also lead to respiratory, gastroenterological or neurological presentations. Immuno-compromised patients such as oncology patients, children with primary immunedeficiencies and those undergoing solid organ and haematopoietic stem cell transplant (HSCT) [1] are at higher risk of developing systemic NTM infections. The incidence of NTM infections is rare but in HSCT patients it is between 50 and 600 times higher than in the general population [2]. A number of risk factors for NTM infections in patients undergoing HSCT have been identified, these include type of graft, conditioning regimen, persistent immunosuppression and prolonged presence of central venous catheter (CVC) [1].

Following HSCT, the most common presentation is central line associated bloodstream infection (CLABSI) (36\%) [2]. Treatment of CVC-related NTM infections can pose problems due to formation of a biofilm on the surface of the CVC which is difficult to eradicate. Prognosis for

Stephanie Habermann

stephanie.habermann1@nhs.net

1 Imperial College NHS Healthcare Trust, London, UK

2 Great Ormond Street Hospital, London, UK
HSCT patients with CVC-related NTM infections is generally good [1].

CLABSI caused by NTM is often caused by water colonisation. A recent systematic review of waterborne NTM hospital outbreaks described 21 distinct outbreaks [3]. All patients affected were susceptible due to a variety of immunodeficiencies or indwelling CVC. Of the 21 outbreaks, 5 occurred in haematology-oncology units and 4 in outpatient clinics. All patients in the haematology-oncology unit outbreaks were immunosuppressed and presented with CLABSI. Only one outbreak occurred on a paediatric unit but did not involve HSCT patients [3]. Here we describe the first outbreak of NTM in a paediatric bone marrow transplant (BMT) unit in England with water contamination of CVC and needle-free connectors (NC) implicated amongst other factors.

This outbreak investigation occurred on the paediatric haematology BMT unit at St Marys Hospital, a teaching hospital in London, between February and September 2010. The unit carries out approximately 35 allogeneic transplants yearly, mostly in children with haemoglobinopathies or red cell disorders.

A case was defined as any paediatric haematology patient with a positive blood culture (BC) for NTM in the 8-month period of the outbreak. Medical records were reviewed to obtain clinical and laboratory data including patient demographics, BMT type, conditioning regimen, presence of CVC and NC, clinical symptoms, positive $\mathrm{BC}$, treatment and outcome. The absence of such cases during the same period the year before, led us to define it as an outbreak, especially as neither the surveillance policy nor the laboratory and blood sampling techniques changed.

In order to identify the potential source of infection, water samples were obtained from the paediatric BMT unit. The infection control practices of the department were 


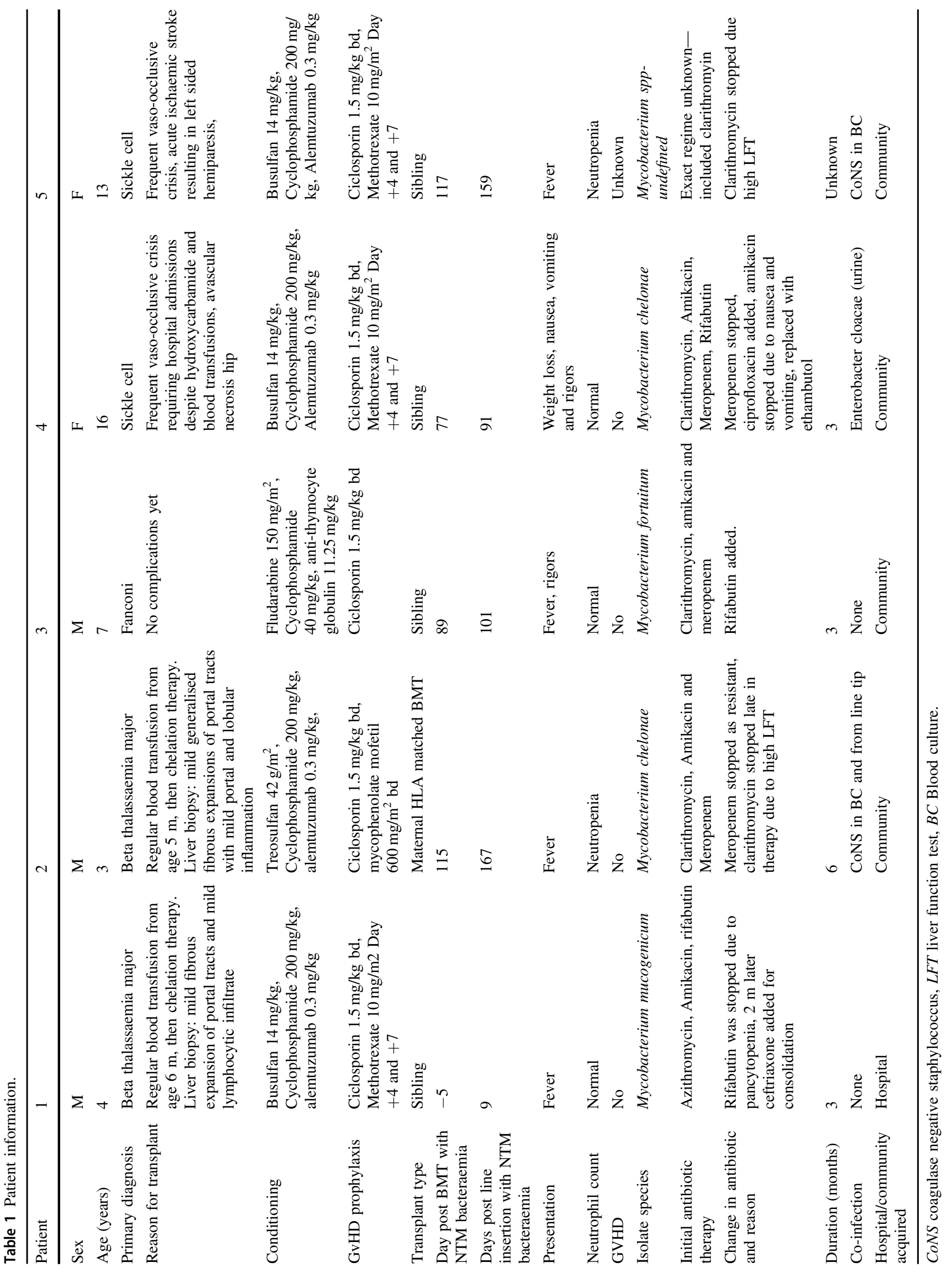


reviewed, with a special emphasis on handling of CVC sites and their $\mathrm{NC}$, as well as the relevant information provided to patients at discharge.

The patient characteristics of the five patients that developed NTM infections are described in Table 1. All of the patients had CVC with $\mathrm{NC}$ which were removed for source control. Three patients required subsequent replacement. All patients were treated with prolonged courses of antibiotics; amendment due to drug adverse events was required for all. No infection recurrence occurred, and all patients made a full recovery.

All patients grew RGM from CVC BCs. The median time from CVC insertion to positive BC was 101 days (range 9-167). The median time from the stem cell infusion to positive BC was 89 days (range -5-117).

Atypical mycobacteria were identified in water samples from the BMT unit. This was discussed with Public Health England who felt that the significance of NTM in water samples was unclear. Subsequently, line care was identified as the main contributing factor to the outbreak. Further qualitative work, including guideline review and patient interviews, identified that patients showered and bathed at home without adequately protecting the $\mathrm{CVC}$, a recognised source of contamination. Therefore, guidelines were modified, and health-workers and patients received further training on handling of CVC and NC. Sterile transparent semi-permeable dressings were provided for patient use when bathing and showering. Finally, the model of NCs was changed. No further NTM infections were detected following these interventions.

We report a cluster of RGM CLABSI in a paediatric BMT unit over an 8-month period. There were multiple factors contributing to this outbreak, and CVC and NC exposure to contaminated tap water was implicated. Updating guidelines and educating patients and health professionals were interventions that contributed to no further cases being identified.

A literature review identified eight previous outbreaks of CLABSI due to RGM [4-11]. A total of 43 patients were affected; 21 were children. All of these outbreaks occurred on haematology-oncology units and half involved HSCT patients $[4,7,8,11]$.

In this outbreak we identified similar RGM to previous outbreaks. However in previous outbreaks most patients were affected by the same organism whereas this outbreak was polymicrobial. A potential explanation is that in this outbreak most patients were outpatients and may have acquired the NTM from tap water at home. Despite this observation, we describe this as an outbreak as all patients were actively managed at one centre.

The presumed mechanism of contamination of the CVC and $\mathrm{NC}$ was with tap water during bathing and showering, when the lines were not adequately covered. Therefore,
CVC handling guidelines were reviewed and NCs changed to a different model, which have been successful strategies in previous similar reports $[4,5,8-11]$. An education programme on CVC care for families was updated and education for healthcare staff refreshed.

In view of the uncertain significance of the water sampling results, polymicrobial results indicating multiple water sources and interventions being successful at preventing further outbreaks, no further action was required to address water source contamination.

In this cluster, all patients underwent CVC removal and treatment with prolonged courses of antibiotics, as recommended in general guidelines for management of mycobacterial CVC-related bacteraemia (14). However, as there are currently no specific guidelines for the treatment of NTM infections in HSCT recipients, treatment is quite variable. All previous outbreaks had CVC removed but only half were managed with prolonged courses of antibiotics $[5,7,9,10]$. Previous reports support short courses of antibiotics, and given the frequent side effects associated with prolonged antimicrobial treatments, shortening treatment of NTM bacteraemia in patients without disseminated disease should be considered. Further research into optimal length of treatment and development of NTM treatment guidelines outside of pulmonary disease would be a step forward in the management of NTM in immunocompromised patients.

In conclusion, this report highlights that NTM should be considered as a cause of fever in immuno-compromised patients with CVC especially as morbidity is high and treatment long and costly. Successful strategies for disease prevention included: increasing awareness of potential sources of infection, including water and $\mathrm{NC}$ and ensuring regular patient and staff education regarding management of CVC and NC.

\section{Compliance with ethical standards}

Conflict of interest The authors declare no competing interests.

Publisher's note Springer Nature remains neutral with regard to jurisdictional claims in published maps and institutional affiliations.

Open Access This article is licensed under a Creative Commons Attribution 4.0 International License, which permits use, sharing, adaptation, distribution and reproduction in any medium or format, as long as you give appropriate credit to the original author(s) and the source, provide a link to the Creative Commons license, and indicate if changes were made. The images or other third party material in this article are included in the article's Creative Commons license, unless indicated otherwise in a credit line to the material. If material is not included in the article's Creative Commons license and your intended use is not permitted by statutory regulation or exceeds the permitted use, you will need to obtain permission directly from the copyright holder. To view a copy of this license, visit http://creativecommons. org/licenses/by/4.0/. 


\section{References}

1. Al-Anazi KA, Al-Jasser AM, Al-Anazi WK. Infections caused by non-tuberculous mycobacteria in recipients of hematopoietic stem cell transplantation. Front Oncol. 2014;4:311.

2. Doucette K, Fishman JA. Nontuberculous mycobacterial infection in hematopoietic stem cell and solid organ transplant recipients. Clin Infect Dis. 2004;38:1428-39.

3. Li T, Abebe LS, Cronk R, Bartram J. A systematic review of waterborne infections from nontuberculous mycobacteria in health care facility water systems. Int $\mathrm{J}$ Hyg Environ Health. 2017;220:611-20.

4. Kline S, Cameron S, Streifel A, Yakrus MA, Kairis F, Peacock K, et al. An outbreak of bacteremias associated with mycobacterium mucogenicum in a hospital water supply. Infect Control Hosp Epidemiol. 2004;25:1042-9.

5. Livni G, Yaniv I, Samra Z, Kaufman L, Solter E, Ashkenazi S, et al. Outbreak of mycobacterium mucogenicum bacteraemia due to contaminated water supply in a paediatric haematologyoncology department. J Hosp Infect. 2008;70:253-8.

6. Cooksey RC, Jhung MA, Yakrus MA, Butler WR, Adekambi T, Morlock GP, et al. Multiphasic approach reveals genetic diversity of environmental and patient isolates of mycobacterium mucogenicum and mycobacterium phocaicum associated with an outbreak of bacteremias at a Texas hospital. Appl Environ Microbiol. 2008;74:2480-7.

7. Wei MC, Banaei N, Yakrus MA, Stoll T, Gutierrez KM, Agarwal R. Nontuberculous mycobacteria infections in immunocompromised patients: single institution experience. J Pediatr Hematol Oncol. 2009;31:556-60.

8. Shachor-Meyouhas Y, Sprecher H, Eluk O, Ben-Barak A, Kassis I. An outbreak of Mycobacterium mucogenicum bacteremia in pediatric hematology-oncology patients. Pediatr Infect Dis J. 2011;30:30-2.

9. Baird SF, Taori SK, Dave J, Willocks LJ, Roddie H, Hanson M. Cluster of non-tuberculous mycobacteraemia associated with water supply in a haemato-oncology unit. J Hosp Infect. 2011;79:339-43.

10. Ashraf MS, Swinker M, Augustino KL, Nobles D, Knupp C, Liles $\mathrm{D}$, et al. Outbreak of mycobacterium mucogenicum bloodstream infections among patients with sickle cell disease in an outpatient setting. Infect Control Hosp Epidemiol. 2012;33:1132-6.

11. Tagashira Y, Kozai Y, Yamasa H, Sakurada M, Kashiyama T, Honda $\mathrm{H}$. A cluster of central line-associated bloodstream infections due to rapidly growing nontuberculous mycobacteria in patients with hematologic disorders at a Japanese tertiary care center: an outbreak investigation and review of the literature. Infect Control Hosp Epidemiol. 2015;36:76-80. 